\title{
Chronic intrathecal cannulation enhances nociceptive responses in rats
}

F.R.C. Almeida, I.R.S. Schivo,

B.B. Lorenzetti and S.H. Ferreira
Departamento de Farmacologia, Faculdade de Medicina de Ribeirão Preto, Universidade de São Paulo, Ribeirão Preto, SP, Brasil

\section{Correspondence \\ S.H. Ferreira \\ Departamento de Farmacologia \\ FMRP, USP \\ Av. Bandeirantes, 3900 \\ 14049-900 Ribeirão Preto, SP \\ Brasil \\ Fax: + 55-16-623-2792 \\ E-mail: shferrei@fmrp.usp.br \\ Research supported by FAPESP \\ and CNPq. \\ The present address of \\ F.R.C. Almeida is Departamento de Bioquímica e Farmacologia, CCS, UFPI, Teresina, PI, Brasil, and that of B.B. Lorenzetti is Departamento de Farmacologia, Setor de Ciências Biológicas, UFPR, Curitiba, PR, Brasil.}

Received November 25, 1999 Accepted May 3, 2000

\section{Abstract}

The influence of a chronically implanted spinal cannula on the nociceptive response induced by mechanical, chemical or thermal stimuli was evaluated. The hyperalgesia in response to mechanical stimulation induced by carrageenin or prostaglandin $\mathrm{E}_{2}\left(\mathrm{PGE}_{2}\right)$ was significantly increased in cannulated $(\mathrm{Cn})$ rats, compared with naive $(\mathrm{Nv})$ or sham-operated (Sh) rats. Only $\mathrm{Cn}$ animals presented an enhanced nociceptive response in the first phase of the formalin test when low doses were used $(0.3$ and $1 \%)$. The withdrawal latency to thermal stimulation of a paw inflamed by carrageenin was significantly reduced in Cn rats but not in Nv or Sh rats. In contrast to Nv and Sh rats, injection in $\mathrm{Cn}$ animals of a standard non-steroid anti-inflammatory drug, indomethacin, either intraperitoneally or into the spinal cord via an implanted cannula or by direct puncture of the intrathecal space significantly blocked the intensity of the hyperalgesia induced by $\mathrm{PGE}_{2}$. Cannulated animals treated with indomethacin also showed a significant inhibition of second phase formalin-induced paw flinches. Histopathological analysis of the spinal cord showed an increased frequency of mononuclear inflammatory cells in the Cn groups. Thus, the presence of a chronically implanted cannula seems to cause nociceptive spinal sensitization to mechanical, chemical and thermal stimulation, which can be blocked by indomethacin, thus suggesting that it may result from the spinal release of prostaglandins due to an ongoing mild inflammation.

\section{Introduction}

A technique for spinal intrathecal injection developed by Yaksh and Rudy (1) consists of the chronic cannulation of the subarachnoid space following puncture of the atlanto-occipital membrane. This technique has been used extensively in behavioral studies of drug effects and of spinal receptors and in the evaluation of spinal mediators involved in inflammatory pain (2-8).

\section{Key words}

- Hyperalgesia

- Spinal sensitization

- Intrathecal cannulation

- Nociceptive response
- Inflammation
During an inflammatory response, prostaglandins are released locally at the periphery as well as at spinal and supraspinal sites (9). Prostaglandins administered intrathecally to rats and mice cause increased behavioral nociceptive responses to mechanical, thermal and chemical stimulation (10-12).

The spinal effect of prostaglandins may contribute to the analgesic action of cyclooxygenase inhibitors. In fact, using the chronically implanted catheter technique, Malmberg 
and Yaksh $(13,14)$ demonstrated an inhibitory effect of non-steroid anti-inflammatory drugs on inflammatory nociception, thereby pioneering the idea that the spinal cord is an important site in the mechanism of the analgesic action of these drugs. Supraspinal sites also partially participate in the mechanism of action of cyclooxygenase inhibitors (15).

Recently, it has been shown that the presence of a catheter in the epidural space produces morphological signs of inflammation (16). Thus, we decided to investigate if the presence of a chronically implanted spinal cannula would cause sensitization to nociception induced by a mechanical, chemical (formalin), or thermal stimulus. We have also determined the antinociceptive effect of a standard non-steroid anti-inflammatory drug, indomethacin, injected systemically (intraperitoneally, ip), via a spinal cannula, or by direct intrathecal puncture in sham (Sh), naive (Nv) or chronically implanted (cannulated, $\mathrm{Cn}$ ) animals.

\section{Material and Methods}

\section{Animals}

Male Wistar rats (280-340 g) were housed in temperature-controlled rooms $\left(23 \pm 2^{\circ} \mathrm{C}\right)$ with water and food ad libitum. All experimental procedures conformed to the IASP guidelines on the use of animals in pain research. Rats were used only once.

Drugs

Drugs and chemicals used were 2\% lidocaine chloridrate (Astra, São Paulo, SP, Brazil), diethylic ether (Reagen, Rio de Janeiro, RJ, Brazil), and eosin (Merck, Wilmington, DE, USA). Carrageenin (marine colloids, Philadelphia, PA, USA), prostaglandin $\mathrm{E}_{2}\left(\mathrm{PGE}_{2}\right.$; Sigma Chemical Co., St. Louis, MO, USA), 37\% formaldehyde (Reagen), 2,2,2-tribromoethanol (Aldrich Chemicals Co., Milwaukee, WI, USA), and hematoxylin (Merck) were administered in saline solution. Indomethacin (Merck) was administered in Tris-buffer.

\section{Chronic intrathecal cannulation}

The implantation procedure was that described by Yaksh and Rudy (1). Briefly, after treatment with indomethacin $(5 \mathrm{mg} / \mathrm{kg}, i p)$ and anesthesia with tribromoethanol (250 $\mathrm{mg} / \mathrm{kg}, i p)$, the animals were mounted in a conventional stereotaxic instrument. After a cutaneous incision, the cisternal membrane was slit and $8.5 \mathrm{~cm}$ of a reduced diameter PE-10 polyethylene tube was introduced. The PE-10 polyethylene tube was connected to a metal cannula through PE-20/PE-190 tubing, which remained outside the space. The metal cannula was attached to neck muscles with a suture. Drug injections were made in a volume of $5 \mu 1$ followed by $5 \mu 1$ of vehicle. The animals were submitted to different tests and treatments seven days after the surgical procedure. The sham-operated animals were submitted to the same procedure except for the insertion of the catheter.

\section{Direct intrathecal administration}

During the mechanical hyperalgesia experiments, the drugs were administered under light ether anesthesia according to the procedure described by Papir-Kricheli et al. (17). After a small incision in the skin, injections were made in a maximum volume of 20 $\mu 1$ between L4 and L5 vertebrae.

During the formalin test, the experimental procedures followed the method described by Mestre et al. (18). Briefly, conscious animals were restrained and an injection was made between L5 and L6. The site of injection was confirmed using a $2 \%$ solution of Pontamine Sky Blue mixed with the drug preparation (maximum volume of $10 \mu \mathrm{l}$ ). Incorrect injections were detected by the presence of the dye in the paravertebral musculature or in the dorsal subcutaneous region. 


\section{Nociceptive methods}

Mechanical hyperalgesia. Our modification of the Randall-Selitto rat paw pressure test was used to measure hyperalgesia (15). In this test, a pressure of $20 \mathrm{mmHg}$ is continuously applied to the hindpaw of the rat until the animal presents a typical freezing reaction (reaction time) characterized by a reduction in escape movements, a change in respiratory frequency and the appearance of dorsal fasciculation. After measurement of the basal reaction time (30-35 s), hyperalgesia was induced by intraplantar injection of carrageenin or $\mathrm{PGE}_{2}$. The intensity of hyperalgesia was quantified as the difference in reaction times (delta reaction time) calculated by subtracting the reaction time measured 1 or $3 \mathrm{~h}$ after administration of the hyperalgesic substance from the control reaction time assessed before injection of the hyperalgesic stimulus. The routes and times of administration of the various drugs are indicated in the figure legends.

Formalin test. This procedure followed that described by Wheeler-Aceto et al. (19). The animals were restrained and $50 \mu \mathrm{l}$ of $0.3-5 \%$ formalin solution was rapidly injected subcutaneously into the dorsal surface of the right hindpaw via a 30 -gauge needle. The animals were placed individually in acrylic chambers and two mirrors were positioned on opposite sides of each chamber to facilitate the observation of the formalin-injected paw. Nociceptive behavior was quantified by counting the number of spontaneous flinches/shakes of the injected paw during phase $1(0-10 \mathrm{~min}$, at 1-min intervals) and phase 2 (10-60 $\mathrm{min}$, at 5-min intervals) in the experiments with different concentrations of formalin, and during phase 1 (1-2 and 5-6 $\mathrm{min}$ ) and phase 2 (10-40 and $40-60 \mathrm{~min})$ in the experiments using indomethacin.

Thermal hyperalgesia. This procedure followed that described by Hargreaves et al. (20). Briefly, the animals were placed in an acrylic chamber and allowed to acclimatize for $5 \mathrm{~min}$ before testing. An infrared radiant heat source was then positioned under the chamber floor directly beneath the hindpaw. Each activation of this infrared source triggered an electronic timer, and when the animal removed the paw both the timer and the heat stimulus were stopped (Plantar Test, Ugo Basile; 20). The withdrawal latency was measured before and after the stimulus and the results are shown as the percent ratio between the withdrawal latency of the injected paw and of the control paw at each observation time.

\section{Histopathological analysis of spinal cord slices}

Following the experimental sessions, animals ( $\mathrm{Nv}$, Sh or $\mathrm{Cn}$ ) were randomly selected and perfused with $60 \mathrm{ml}$ of $10 \%$ formaldehyde solution via the right ventricle. The spinal cords were removed carefully and left in formaldehyde for 20-30 days before embedding in paraffin. Serial 5-mm sections were cut from each level of the spinal cord, rehydrated, stained with hematoxylin and eosin and mounted for light microscopy analysis. The number of spinal cords studied was 43, 27 and 25 for Cn, Sh and Nv animals, respectively. The results are reported as percent of the animals whose sections presented mononuclear infiltrate.

\section{Results}

The data in Figure 1 show a dose-dependent mechanical hyperalgesia $(\mathrm{P}<0.05$, ANOVA) in chronically cannulated, naive and sham-operated animals following intraplantar injections of either carrageenin (measured 1 and $3 \mathrm{~h}$ after the challenge; panels $\mathrm{A}$ and $\mathrm{B}$, respectively) or $\mathrm{PGE}_{2}$ (measured $3 \mathrm{~h}$ after the challenge; panel $\mathrm{C}$ ).

Cannulation resulted in increased magnitude of hyperalgesia. Thus, a significant increase in the intensity of hyperalgesia for lower doses of carrageenin (2.5-10 $\mu \mathrm{g})$ or 


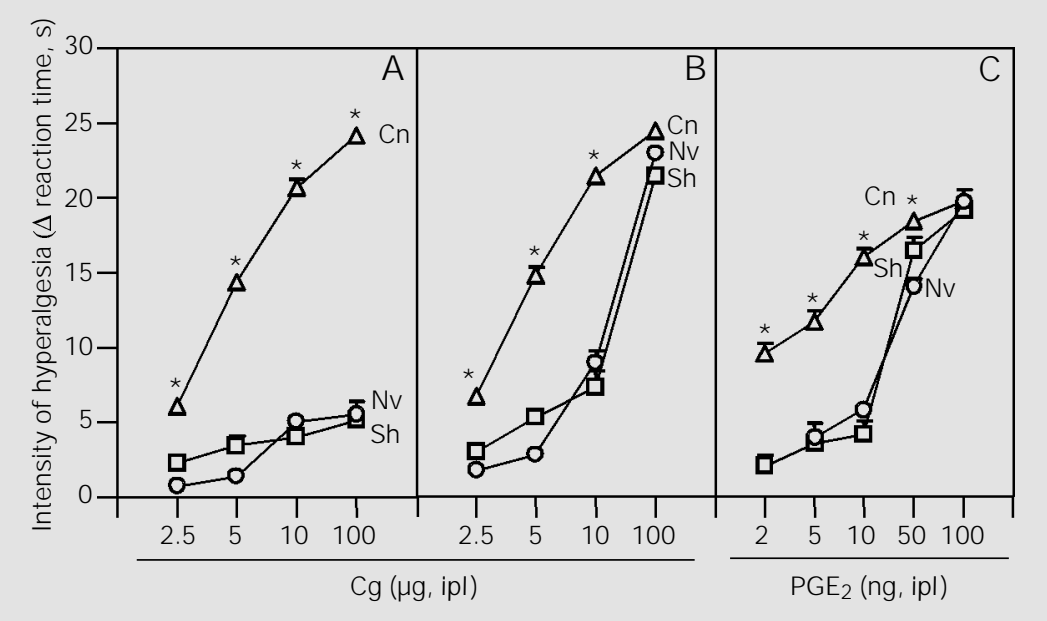

Figure 1 - Comparison of the intensity of mechanical hyperalgesia in response to increasing doses of carrageenin $(\mathrm{Cg})$ or $\mathrm{PGE}_{2}$ in chronically cannulated ( $\mathrm{Cn}$, triangles), naive ( $\mathrm{Nv}$, circles) and sham-operated (Sh, squares) rats. The intensity of hyperalgesia was measured $1 \mathrm{~h}$ (panel A) and $3 \mathrm{~h}$ (panel B) after the different doses of carrageenin (2.5-100 $\mu \mathrm{g}$, intraplantar (ipl)). Panel $\mathrm{C}$ shows the intensity of hyperalgesia measured $3 \mathrm{~h}$ after an ipl injection of $\mathrm{PGE}_{2}(5-100 \mathrm{ng} / \mathrm{ml})$. The data (mean \pm SEM for five animals per group) were analyzed by one-way ANOVA followed by Duncan's test. *P<0.05 compared to Nv and Sh animals.

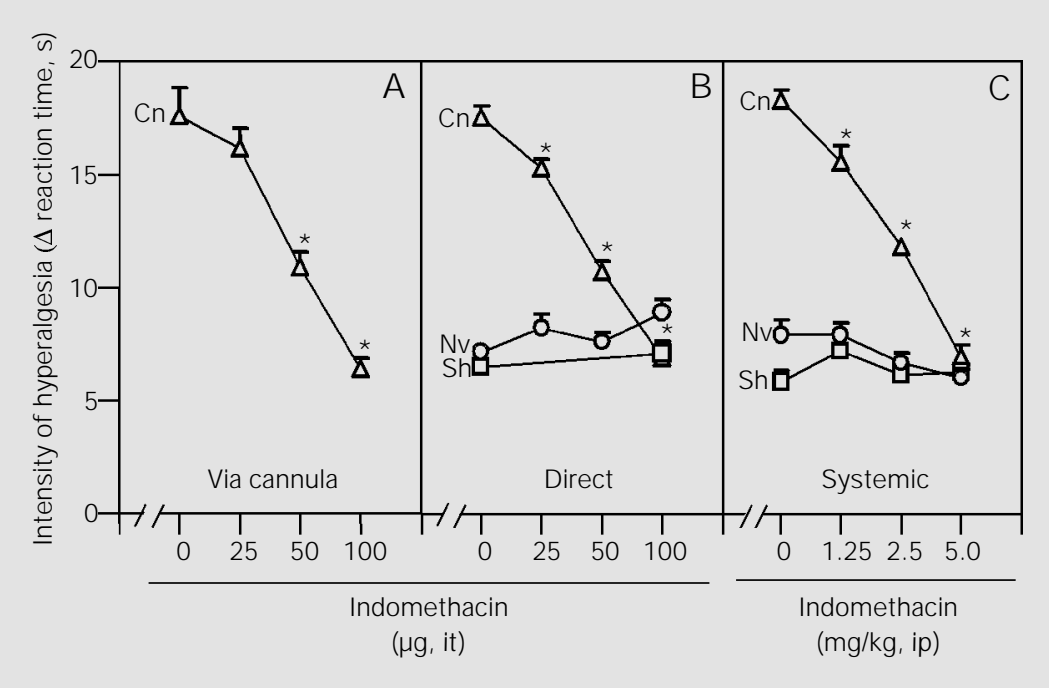

Figure 2 - Effect of indomethacin on the intensity of $\mathrm{PGE}_{2}$ hyperalgesia in chronically cannulated ( $\mathrm{Cn}$, triangles), sham-operated (Sh, squares) and naive (Nv, circles) animals. Indomethacin was administered via an intrathecal (it) cannula (panel A), directly into the subarachnoid space (panel B) or systemically (ip, panel C) 30 min before intraplantar injection of $\mathrm{PGE}_{2}(10 \mathrm{ng} / \mathrm{paw})$. Each point represents the mean reaction time (s) $\pm \mathrm{SEM}$ of 513 animals per group measured $3 \mathrm{~h}$ after the hyperalgesic challenge. The data were analyzed by one-way ANOVA followed by Duncan's test (asterisks indicate significant differences for each dose, $\mathrm{P}<0.05)$.
$\mathrm{PGE}_{2}(2-10 \mathrm{ng})$ was observed in $\mathrm{Cn}$ when compared with $\mathrm{Nv}$ or Sh animals $(\mathrm{P}<0.05$, Duncan's test). In contrast with $\mathrm{Nv}$ or Sh animals, for $\mathrm{Cn}$ animals there were no significant differences between the curves measured at one and $3 \mathrm{~h}$ after carrageenin injections ( $\mathrm{P}>0.05$, ANOVA; comparing panels $A$ and $B$ ). The greater sensitivity of $\mathrm{Cn}$ animals is also illustrated by the fact that the hyperalgesic plateau was reached within $1 \mathrm{~h}$ as compared with $\mathrm{Nv}$ or $\mathrm{Sh}$ animals (comparing panel A with B, $100 \mu \mathrm{g}$ of carrageenin). This figure also shows that there was no significant difference between the hyperalgesic effect induced in $\mathrm{Nv}$ and $\mathrm{Sh}$ animals. The delta reaction times for $\mathrm{Cn}, \mathrm{Sh}$ or Nv animals measured before the hyperalgesic paw treatment with $\mathrm{PGE}_{2}$ or carrageenin did not differ significantly (data not shown).

Figure 2 shows that the increased $\mathrm{PGE}_{2}-$ induced hyperalgesic effect on $\mathrm{Cn}$ animals was dose-dependently blocked by indomethacin injected via a cannula (panel $\mathrm{A}=25-100$ ng), directly into the intrathecal space (panel $\mathrm{B}=25-100 \mathrm{ng}$ ), or systemically (panel $\mathrm{C}=$ $1.25-5 \mathrm{mg} / \mathrm{kg}$, ip) and that the drug inhibited in a dose-dependent manner the hyperalgesic effect of $10 \mathrm{ng} / \mathrm{paw}$ of $\mathrm{PGE}_{2}(\mathrm{P}>0.05$, ANOVA), an effect that was significantly different for Nv or Sh animals. These results were obtained $3 \mathrm{~h}$ after intraplantar $\mathrm{PGE}_{2}$ challenge because the hyperalgesic response reached a plateau only at this time. This figure also shows that intraperitoneal or direct intrathecal injections of indomethacin did not affect the relatively small intensity of the hyperalgesic effect induced by $\mathrm{PGE}_{2}$ in $\mathrm{Sh}$ and $\mathrm{Nv}$ animals (no difference between doses, $\mathrm{P}>0.05$ ).

In the second series of experiments, formalin was used as a chemical stimulator, inducing a dose-dependent number of flinches (first phase, $0-10 \mathrm{~min} ; \mathrm{P}<0.05$, ANOVA; Figure 3). This dose dependency was also observed in the second phase of the test (10-60 min; data not shown). Figure 3 
shows that $\mathrm{Cn}$ rats presented a higher frequency of flinches after stimulation with 0.3 and $1 \%$ of formalin when compared with $\mathrm{Nv}$ and Sh animals ( $\mathrm{P}<0.05$, Duncan's test $)$. An increase in the number of flinches in $\mathrm{Cn}$ animals was also observed in the second phase (data not shown). Dorsal injections of $5 \%$ formalin did not cause significant differences between the three groups of animals during the first phase but did in the second phase of the test (comparing controls of panel $\mathrm{B}$ with $\mathrm{A}$ and $\mathrm{C}$ with $\mathrm{A}$ of Figure 4).

Figure 4 also shows that the number of flinches observed during the second phase of the formalin test ( $5 \%$ formalin) was significantly inhibited when indomethacin was administered to chronically implanted animals either directly into the intrathecal space or via the cannula (panels $\mathrm{B}$ and $\mathrm{C}$, respectively; $\mathrm{P}<0.01$ and $\mathrm{P}<0.001$, unpaired Student $t$-test). Note that administration of indomethacin $(10 \mu \mathrm{g})$ by direct puncture did not significantly affect flinching in Nv animals when compared with the control group (panel A; Tris-buffer).

In the third series of experiments, using thermal stimulation of the paw inflamed with increasing doses of carrageenin (31-125 $\mu \mathrm{g})$, the $\mathrm{Cn}$ animals showed a significant sensitization $1 \mathrm{~h}$ after carrageenin challenge $(\mathrm{P}<0.05$, one-way ANOVA followed by Duncan's test), compared with $\mathrm{Nv}$ or $\mathrm{Sh}$ animals (Figure 5). This differential sensitization was no longer significant $3 \mathrm{~h}$ after carrageenin challenge, when paw edema reached maximal values (data not shown). Note that there were significant differences between the $\mathrm{Cn}$ controls and the $\mathrm{Nv}$ or $\mathrm{Sh}$ animals (C).

No inflammatory cells were found in sections of spinal cords from $\mathrm{Nv}$ animals $(\mathrm{N}=$ $25)$. The presence of predominantly mononuclear inflammatory cell infiltration was observed in the spinal cords of 20 and 69\% of Sh $(\mathrm{N}=27)$ and $\mathrm{Cn}$ animals $(\mathrm{N}=43)$, respectively.

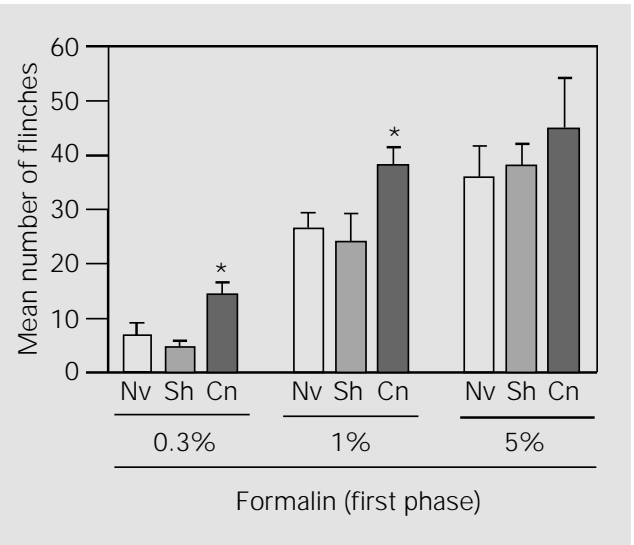

Figure 3 - Differences in the intensity of response of chronically cannulated $(\mathrm{Cn})$, naive $(\mathrm{Nv})$ and sham-operated (Sh) rats during phase 1 of the formalin test. Formalin was administered at different doses of $0.3,1$, and $5 \%$. Each bar is the mean \pm SEM number of flinches of 6-12 animals, measured for $10 \mathrm{~min}$ after the nociceptive stimulus. Data were analyzed by one-way ANOVA followed by Duncan's test. $* \mathrm{P}<0.05$ compared to normal or Sh animals.

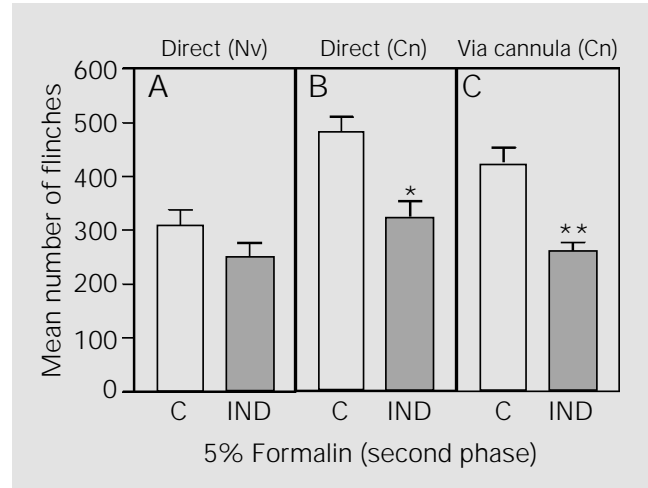

\section{Discussion}

In our first series of experiments with $\mathrm{PGE}_{2}$ and carrageenin, there was an increased hyperalgesic response to paw pressure in $\mathrm{Cn}$ animals compared with $\mathrm{Nv}$ or Sh animals (Figure 1). This increased sensitivity of $\mathrm{Cn}$ animals could be noted either by an enhancement of the effect of lower doses of carrageenin or by an early hyperalgesic plateau. $\mathrm{Cn}$ animals reached the plateau $1 \mathrm{~h}$ after intraplantar injection of carrageenin while 3 $\mathrm{h}$ were necessary for Nv or Sh animals (compare A and B in Figure 1). In Cn animals carrageenin caused an earlier plateau effect when compared with $\mathrm{PGE}_{2}$ (data not shown). This might result from the fact that, in addition to $\mathrm{PGE}_{2}$, carrageenin releases prostacyclin and this eicosanoid causes a much earlier response and plateau than that induced by $\mathrm{PGE}_{2}(21)$.
Figure 4 - Inhibitory effect of indomethacin on the increased response of the second phase (10-60 $\mathrm{min}$ ) of the $5 \%$ formalin test in cannulated $(\mathrm{Cn})$ animals. Panels A and B show the effects of direct injections of indomethacin (IND, $10 \mu \mathrm{g} / 10 \mu \mathrm{l}$ ) administered $30 \mathrm{~min}$ before subcutaneous injection of formalin to naive (Nv) and $\mathrm{Cn}$ animals. Panel $\mathrm{C}$ shows the effect of IND administration through a cannula. The controls (C) received $10 \mu$ l of Tris-buffer. The bars represent the mean \pm SEM number of flinches of the injected paw of 9-12 animals per group. The asterisks indicate significant differences $(* P<0.01$ and $* * \mathrm{P}<0.001$, unpaired Student t-test). 
An increased response was also observed after chemical stimulation. $C n$ rats presented a higher frequency of flinches in response to 0.3 and $1 \%$ formalin during the first phase of the test when compared with $\mathrm{Nv}$ and $\mathrm{Sh}$ animals (Figure 3 ). The presence of the cannula increased the number of flinches in response to $5 \%$ formalin in the second phase compared with control Nv animals (Figure 4).

The hypersensitivity to thermal stimulation in paws inflamed by increasing doses of carrageenin was significantly higher in $\mathrm{Cn}$ animals than in Nv or Sh animals (Figure 5). However, no significant differences were observed in the controls not sensitized with intraplantar administration of carrageenin.

Thus, the common denominator observed with different experimental methods of inflammatory nociception was an increased responsiveness of the chronically cannulated animals to mechanical, chemical or thermal stimuli. It should be noted, however, that when mechanical (results not shown) and thermal (Figure 5) stimuli were used there was no difference between control paws of $\mathrm{Nv}$ and $\mathrm{Cn}$ animals. Thus, the presence of the cannula did not produce of itself a detectable increase in nociception in tests using pressure or thermal stimuli. However, in $\mathrm{Cn}$ animals the inflammation occurring when hyperalgesia was induced by formalin (which

Figure 5 - Comparison of cannulated (Cn), naive ( $\mathrm{Nv}$ ) and sham (Sh) animals in terms of withdrawal latencies of carrageenin (Cg)-inflamed rat paws in response to thermal stimulation. The bars represent groups injected with different doses of carrageenin (intraplantar, ipl). C is the control group injected with saline. The results are the mean \pm SEM percent ratio between carrageenin-injected and control paws $1 \mathrm{~h}$ after injection of the stimulus in 5-13 animals per group. Data were analyzed by ANOVA followed by Duncan's test. The asterisks indicate significant differences between $\mathrm{NV}$ and $\mathrm{C} n$ animals $(\mathrm{P}<0.05)$. with a late plateau) or $\mathrm{PGE}_{2}$ (which mimics inflammatory hyperalgesia) caused significantly higher nociceptive responses than observed in Sh or Nv animals. We observed no differences in the nociceptive response of the control $\mathrm{Cn}$, Sh or Nv animals before the hyperalgesic treatment either in the paw pressure test (data not shown) or when the paw was thermally stimulated (Figure 5). Thus, it appears that sensitization of the primary sensory neuron is a condition necessary for detection of the hyperalgesic effect of the implanted cannula. A possible reason for the failure of the mechanical and thermal nociception test in detecting the presence of the cannula may be the low sensitivity of the test. Alternatively, and more plausibly, it may be that the lowering of the threshold of the primary sensory neurons induced by carrageenin, $\mathrm{PGE}_{2}$ or formalin is a necessary condition for detection of the supraspinal and/or spinal sensitization provoked by the presence of the spinal cannula. This interpretation explains why a blockade of spinal neuron sensitization by intrathecal administration of indomethacin inhibited in a dosedependent manner the hyperalgesia induced by intraplantar injection of $\mathrm{PGE}_{2}$ in the pressure test (Figure 2) or by carrageenin in the thermal test (Figure 5). The blockade of $\mathrm{PGE}_{2}$ paw hyperalgesia by intrathecally administered indomethacin is noteworthy because this effect is not blocked by systemic or intraplantar treatment with indomethacin (21).

Infection is not a usual complication in studies using the intrathecal cannulation procedure. However, there are reports of altered responses to drug administration with an acute or chronic implantation of a catheter $(16,22-24)$. Inflammatory changes along the catheter tract have been observed, as well as spinal cord compression in some cases (16). In fact, mononuclear cell infiltration was frequently found in cannulated animals, compared with absence of infiltration in Nv animals. It should be pointed out that, as previ- 
ously described, the presence of migrating cells in an injured tissue is not a determinant condition for induction of hyperalgesia (25). The presence of mononuclear cells in a number of Sh animals (which was not observed in Nv animals) may indicate that mere puncture of the occipital membrane may induce a mild inflammatory reaction. Although the presence of cells per se does not indicate sensitization, in order to avoid this possibility, we used Nv animals to replicate Malmberg and Yaksh's observation of the inhibitory effect of the non-steroid anti-inflammatory drug indomethacin on the second phase of the formalin test at the dose of $5 \%(13,14)$. Indomethacin administration either via a cannula or directly by intrathecal puncture to chronically cannulated animals caused antinociception. On the other hand, direct intrathecal injection of indomethacin had no significant antinociceptive effect on $\mathrm{Nv}$ animals (Figure 4). Thus, the presence of the cannula permitted the detection of the antinociceptive effect of indomethacin in $\mathrm{Cn}$ but not in Nv animals.

The presence of a chronically implanted cannula (Cn group) also enhanced the sensitivity to otherwise ineffective intraplantar doses of $\mathrm{PGE}_{2}(10 \mathrm{ng} / \mathrm{paw}$ in $\mathrm{Nv}$ and $\mathrm{Sh}$ animals) and in such instances, the administration of indomethacin (via cannula, directly by spinal puncture or systemically) blocked this prostaglandin effect in a dosedependent manner(Figure 2). In a pilot study with a higher dose of $\mathrm{PGE}_{2}(100 \mathrm{ng} / \mathrm{paw})$, administration of indomethacin either intraperitoneally $(5 \mathrm{mg} / \mathrm{kg})$ or via an intrathecal cannula $(50 \mu \mathrm{g})$ or directly into the spinal cord $(50 \mu \mathrm{g})$ did not modify the magnitude of hyperalgesia (data not shown). It should be pointed out that this high $\mathrm{PGE}_{2}$ dose already produces a maximal hyperalgesia in response to pressure when injected into Nv animals. Thus, the lack of effect of indomethacin might reflect the fact that abolition of spinal sensitization by the cannula does not modify the maximal effect of $\mathrm{PGE}_{2}$. The intrathecal indomethacin blockade of nociceptive behavior induced by mechanical or thermal stimulation of hyperalgesic paws may be due to the ongoing sensitization of the spinal sensory neurons caused by the presence of the cannula in the spinal cord and may therefore reflect an inhibition of the formation of prostaglandins due to a mild inflammation (small presence of neutrophils) induced by the chronically implanted cannula. The sensory neurons sensitized by spinal cannulation are situated above the lumbar region, probably in the thoracic region (the distal tip of the cannula approaches the lumbar region). Supporting this suggestion is the fact that the fluid of the direct intrathecal injections bathes this region without reaching the cervical vertebrae (Ferreira SH, personal observations).

We have previously reported that intrathecal administration of $\mathrm{PGE}_{2}$ (L4-L5) causes a retrograde sensitization of the primary sensory neuron (via glutamate release) inducing hyperalgesia in both paws $(26,27)$. In our experiments, however, the presence of the intrathecal cannula did not change the sensitivity of the paws, indicating that the enhancement of the nociceptive response observed is mainly due to sensitization of spinal neurons. The significance of this finding is that, for certain experimental protocols, the central antinociceptive effect of non-steroid anti-inflammatory drugs can be induced by the presence of a chronically implanted cannula. On the other hand, our results clearly indicate the crucial role of sensitization of primary sensory neurons in the induction of nociceptive behavior. This idea implies that in chronic pain, in addition to possible spinal mechanisms of sensitization (28), a lowering of the threshold of primary sensory neurons may also be necessary. Thus, analgesics that promote an effective and prolonged blockade of peripheral inflammatory hyperalgesia without the secondary effects of steroid and non-steroid antiinflammatory drugs continue to represent an important target for drug development. 


\section{References}

1. Yaksh TL \& Rudy TA (1976). Chronic catheterization of the spinal subarachnoid space. Physiology and Behavior, 17: 13571358.

2. Yaksh TL \& Rudy TA (1976). Analgesia mediated by a direct spinal action of narcotics. Science, 192: 1357-1358.

3. Malmberg $A B \&$ Yaksh TL (1993). Pharmacology of the spinal action of ketorolac, morphine, ST-91, U50488H, and L-PIA on the formalin test and an isobolographic analysis of the NSAID interaction (see comments). Anesthesiology, 79: 270-281.

4. Malmberg AB \& Yaksh TL (1995). Cyclooxygenase inhibition and the spinal release of prostaglandin E2 and amino acids evoked by paw formalin injection: a microdialysis study in unanesthetized rats. J ournal of Neuroscience, 15: 2768-2776.

5. Malmberg AB \& Yaksh TL (1995). The effect of morphine on formalin-evoked behaviour and spinal release of excitatory amino acids and prostaglandin E2 using microdialysis in conscious rats. British J oumal of Pharmacology, 114: 1069-1075.

6. Akerman B, Rosell S \& Folkers K (1982). Intrathecal (D-Pro2, D-Trp7,9)-SP elicits hypoalgesia and motor blockade in the rat and antagonizes noxious responses induced by substance P. Acta Physiologica Scandinavica, 114: 631-633.

7. Yamamoto T \& Yaksh TL (1993). Effects of intrathecal strychnine and bicuculline on nerve compression-induced thermal hyperalgesia and selective antagonism by MK-801. Pain, 54: 79-84.

8. Chaplan SR, Pogrel J W \& Yaksh TL (1994). Role of voltage-dependent calcium channel subtypes in experimental tactile allodynia. J ournal of Pharmacology and Experimental Therapeutics, 269: 1117-1123.

9. Ramwell PW, Shaw J E \& J essup R (1966). Spontaneous and evoked release of prostaglandins from frog spinal cord. American J ournal of Physiology, 211: 998-1004.

10. Ferreira SH (1983). Peripheral and central analgesia. In: Bonica JJ, Lindblom U \&
Iggor A (Editors), Advances in Pain Research and Therapy. Raven Press, New York.

11. Taiwo YO \& Levine J D (1986). Indomethacin blocks central nociceptive effects of PGF2 alpha. Brain Research, 373: 81-84.

12. Minami $T$, Uda $R$, Horiguchi $S$, Ito $S$, Hyodo M \& Hayaishi O (1994). Allodynia evoked by intrathecal administration of prostaglandin E2 to conscious mice. Pain, 57: 217-223.

13. Malmberg AB \& Yaksh TL (1992). Antinociceptive actions of spinal nonsteroidal anti-inflammatory agents on the formalin test in the rat. J ournal of Pharmacology and Experimental Therapeutics, 263: 136146.

14. Malmberg AB \& Yaksh TL (1992). Hyperalgesia mediated by spinal glutamate or substance $P$ receptor blocked by spinal cyclooxygenase inhibition. Science, 257: 1276-1279.

15. Ferreira SH, Lorenzetti BB \& Correa FM (1978). Central and peripheral antialgesic action of aspirin-like drugs. European J ournal of Pharmacology, 53: 39-48.

16. Serpell MG, DeLeo JA, Coombs DW, Colburn RW, Twitchell BB, Willenbring $S$ \& Fromm C (1993). Intrathecal catheterization alone reduces autotomy after sciatic cryoneurolysis in the rat. Life Sciences, 53: 1887-1892.

17. Papir-Kricheli D, Frey J , Laufer R, Gilon C, Chorev M, Selinger Z \& Devor M (1987). Behavioural effects of receptor-specific substance $P$ agonists. Pain, 31: 263-276.

18. Mestre C, Pelissier T, Fialip J , Wilcox G \& Eschalier A (1994). A method to perform direct transcutaneous intrathecal injection in rats. J ournal of Pharmacology and Toxicology Methods, 32: 197-200.

19. Wheeler-Aceto $H$, Porreca $F \&$ Cowan $A$ (1990). The rat paw formalin test: comparison of noxious agents. Pain, 40: 229238.

20. Hargreaves K, Dubner R, Brown F, Flores $C \&$ J oris J (1988). A new and sensitive method for measuring thermal nociception in cutaneous hyperalgesia. Pain, 32: 77-88.

21. Ferreira $\mathrm{SH}$, Nakamura $\mathrm{M} \&$ Abreu Castro MS (1978). The hyperalgesic effects of prostacyclin and prostaglandin E2. Prostaglandins, 16: 31-37.

22. Long J B, Mobley WC \& Holaday JW (1988). Neurological dysfunction after intrathecal injection of Dynorphin A (1-13) in the rat. I. Injection procedures modify pharmacological responses. J ournal of Pharmacology and Experimental Therapeutics, 246: 1158-1166.

23. Stevens CW \& Yaksh TL (1986). Dynorphin $A$ and related peptides administered intrathecally in the rat: A search for putative kappa opiate receptor activity. $J$ ournal of Pharmacology and Experimental Therapeutics, 238: 833-838.

24. Bianchi M, Dib B \& Panerai AE (1998). Interleukin-1 and nociception in the rat. J ournal of Neuroscience Research, 53: 645-650.

25. Castro MSA \& Ferreira SH (1979). Cell migration and hyperalgesia: A paradoxical effect of endotoxin. In: Weissmann G, Samuelsson B \& Paoletti R (Editors), Advances in Inflammation Research. Raven Press, New York.

26. Ferreira SH \& Lorenzetti BB (1994). Glutamate spinal retrograde sensitization of primary sensory neurons associated with nociception. Neuropharmacology, 33: 1479-1485.

27. Ferreira SH \& Lorenzetti BB (1996). Intrathecal administration of prostaglandin E2 causes sensitization of the primary afferent neuron via the spinal release of glutamate. Inflammation Research, 45: 499-502.

28. Coderre TJ, Katz J, Vaccarino AL \& Melzack R (1993). Contribution of central neuroplasticity to pathological pain: review of clinical and experimental evidence. Pain, 52: 259-285. 